\title{
Exploring the Contrast Transitions of Korean Vowels: An Information-theoretic View*
}

Ponghyung Lee

(Daejeon University)

\begin{abstract}
Lee, Ponghyung. (2017). Exploring the Contrast Transitions of Korean Vowels: An Information-theoretic View. Language Research, 53.1, 3-21.
\end{abstract}

\begin{abstract}
What present study purports is to explore two kinds of contrast transitions occurring to Korean vowel inventory, i.e. diphthongization of front rounded vowels $/ \mathrm{y} /$ and $/ \varnothing /$ and merger of front mid-vowels /e/ and $/ \varepsilon /$. Overall, our endeavors start from the premise that the weak contrast associated with concerned vocalic segments might bring about the wavering of what they sound like. The null hypothesis is that the correlates derived from a certain amount of information as a measure of communicative efficiency deeply impact the determination of the degree of contrast among phonological units. For the purpose, relying on the corpora based on written and spoken texts, and other subsidiary data sources, the intervention of surprisal, entropic contribution, relative contrastiveness, and perceptual distance is examined as part of diagnostics on the segmental changes over time. What we found therein is that for a proper explication of $/ y /-/ \varnothing /$ disintegration and $/ \mathrm{e} /-/ \varepsilon /$ merger, the Information-theoretic approach is compelling to a certain extent.
\end{abstract}

Keywords: Korean contrast, Information Theory, /y/-/ø/ disintegration, $/ e /-/ \varepsilon /$ merger

* Thanks to audiences at the Phonology Forum 2016 organized by the Phonological Society of Japan at Kanazawa, Ishikawa Prefecture, Japan on August 24-26, 2016 and the Commemorative Forum Dedicated to the 40th Anniversary of the Linguistic Society of Korea at Seoul National University on January 14-15, 2016, particularly to Toshiyuki Tabata, Timothy Vance, Jeonglim Han for their comments and encouragements on this article. 


\section{Introduction}

The sound pattern of Korean vowels is observed to have undergone significant changes over the past five decades. Aside from plenty of disparities among dialects, these developments follow two strands. Above all, the front rounded vowels $/ \mathrm{y} /$ and $/ \varnothing /$ are fading away, in spite of the prescriptive efforts made by public school textbooks or newscasters and the like. When focusing on the casual speech, it is generally agreed that the marked vowels /y/ and /ø/ were already being lost amongst the younger generations. To be precise, those two sounds disintegrate into /wi/ and /we/, respectively, instead of vanishing without traces (cf. W Hur 1965; J-R Oh 1996; J-Y Shin 2000, among others). Another sort of vowel changes affects the mid-front vowels /e/ and $/ \varepsilon /$, which are less than optimal in light of segmental contrast. Cross-linguistically, the two vowels at hand seem to be awkward bedfellows: e.g. in French /e/ and / $\varepsilon /$ are contrastive only in a final open syllable like lait 'milk' vs. allez 'go present tense'.1) Otherwise, the occurrence is predictable in that $/ \mathrm{e} /$ appears in open syllable and $/ \varepsilon /$ in close syllable. In this sense, Trubetzkoy (1939/1969: 78) dubs the /e/-/ $/$ / distinction as neutralizable, while others like /i/-/e/ as constant. To mitigate the perceptual disadvantages, for instance, English takes an enhancement strategy by diphthongizing /e/ into /eI/, which is true to the vast majority of American and British English, as Labov et al. (2006) and Cruttenden (2001) put forth. Obviously, the alternations through diphthongization contribute to drastic changes in phonetic properties like formant frequencies and duration, which are believed to increase the perceptual distance between concerned front mid-vowels one way or another. In contrast, Korean speakers make use of a segmental merger, as acoustically borne out by the previous literature like H-Y Hwang and S-J Moon (2005) and Y-G Han (2000), to name just a few. In the wake of the two kinds of vowel changes, i.e. the break-up of front rounded vowels $/ \mathrm{y} /$ and $/ \varnothing /$, and amalgamation of front mid-vowels /e/ and $/ \varepsilon /$ into $/ \mathrm{e} /$, current Korean is drifting towards seven vowel system as plotted in Figure 1b, away from the earlier

1) Notice that in general, the word-final obstruent consonants fail to surface in French. 
ten vowel system given in Figure 1a:

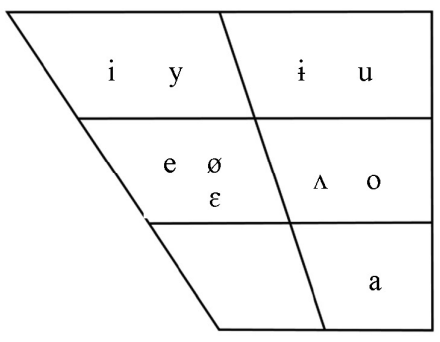

Figure 1. a. Past ten vowel system

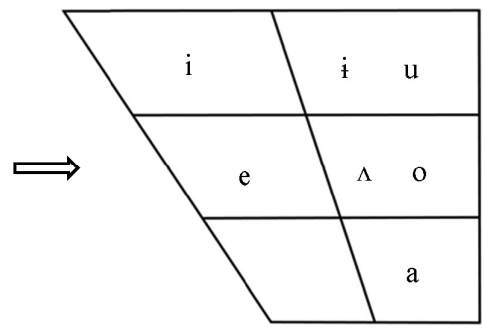

b. Current seven vowel system.

This paper is organized as follows: Section 2 addresses the method pursued in exploring the factors that affect the concerned segmental system. To this end, analytic tools and concepts fundamental to the Information-theoretic approaches will be introduced. Next, the data sources are made clear, trying to define the corpora and other subsidiary databases probed for the current study. Section 3 unveils the investigated results, and delves into the issue of whether the Information-based approach is proper to account for what takes place to Korean vocalic contrast or not. Section 4 sums up our findings and concludes this study.

\section{Method}

2.1. Beyond acoustic correlates alone

By now, the observation that acoustic correlates of concerned sounds and their phonological properties are more often than not at odds with each other is well documented. For instance, formant frequencies of vowel and voice onset time (VOT) of obstruent consonants prove not to be effective in predicting the pattern of loanword adaptation, as noted by LaCharité and Paradis (2005): 

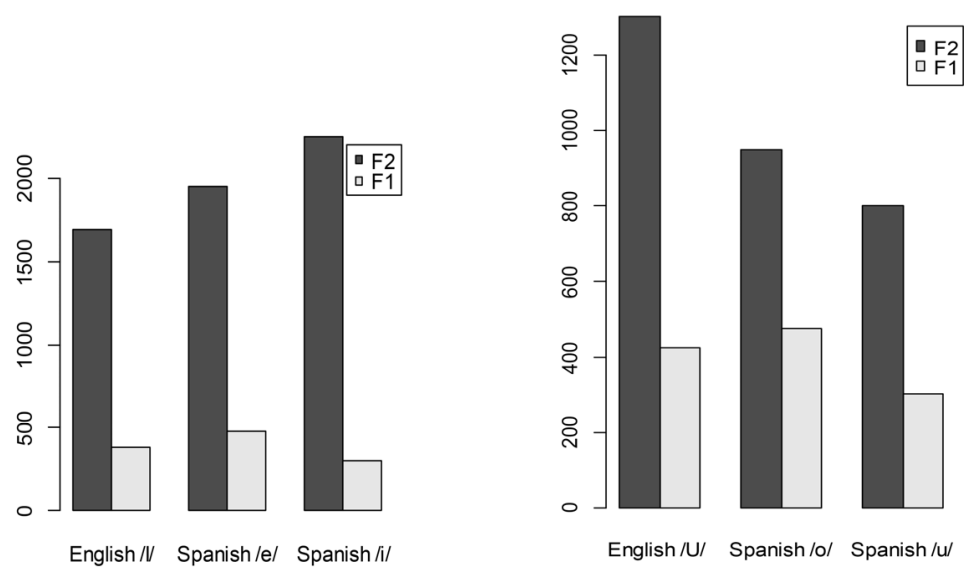

Figure 2. Formants of English and Spanish vowels (n.b. /I/ and /U/ signify IPA /I/ and / /, respectively; based on the measurements by LaCharité and Paradis 2005).

As plotted in Figure 1, the formant frequencies of Spanish /e/ and /o/ are closer to those of English /I/ and / / than those of /i/ and /u/. However, English /I/ and / / / are known to be consistently adapted into Spanish /i/ and /u/, instead of /e/ and /o/. In parallel, the same thing happens to consonants. The range of voice onset time (VOT) of Spanish voiceless stops is precisely congruous to that of English voiced ones by $0-30$ milliseconds. Yet, Spanish speakers adapt English voiceless stops, whose VOT tends to exceed 50 milliseconds, into their voiceless correspondents. In the same vein, the incongruity of the acoustic correlates and perception in English, as pointed out by Hillenbrand et al. (1995), further undermines the claim that the formant frequencies of a vowel solely serve as a reliable means of vowel contrast, as given in Table 1:

Table 1. A Wilcoxon signed rank test for the formants of English vowel pairs $\{\varepsilon-æ\}$ and $\{0-a\}$ (based on the measurements of Hillenbrand et al. 1995)

\begin{tabular}{c|c|c|c|c}
\hline vowel pair & V value & p-value & confusion rate & merger dialect \\
\hline \hline$\varepsilon-æ$ & 43 & 0.7836 & 0.056 & not reported \\
\hline ग- a & 72 & 0.0068 & 0.138 & US Western cot-caught merger \\
\hline
\end{tabular}


The results of the Wilcoxon signed rank test show that the difference in formants 1 through 4 between $/ \varepsilon /$ and $/ æ /$ is not significant as preeminently evidenced by the p-value 0.7836 , while the difference between $/ \mathrm{s} /$ and $/ a /$ is definitely significant, confirmed by the minuscule $p$-value 0.0068 . However, those acoustic results squarely contradict the perceptual outcomes, as Hillenbrand et al. (1995) found out, that the pair / $\varepsilon-æ /$ proves to show less confusion rates to American English speakers than /ว- $\mathrm{a}$ / by the rate of 0.056 to 0.138 , as made explicit in Figure 2:

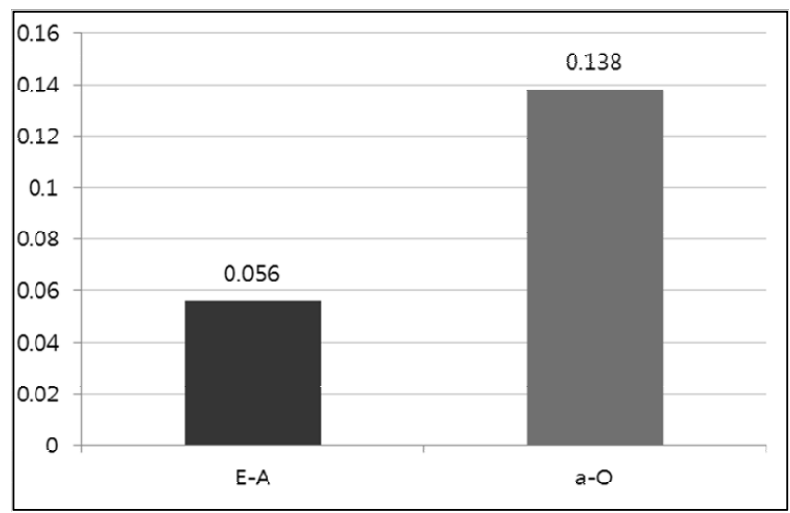

Figure 3. The confusion rates of English vowels pairs $/ \varepsilon-æ /$ and $/ a-$ ऽ/ (measured by Hillenbrad et. al 1995; n.b. the symbols E, A, a, O signify IPA $/ \varepsilon /, / æ /, / a /$, and /っ/, respectively).

Notice that the acoustic disadvantages that the pair $/ \varepsilon-æ /$ suffers hardly brings about the sort of changes occurring to the pair / $a-\supset /$, which is called cot-caught merger. The vowel meger is prevalent in the Western and Midwestern American dialects, as noted by Labov et al. (2006).

To conclude, what we found on the acoustic properties associated with a sound is enough to lead us to be skeptical about the status of fragmented acoustic properties as a means of predicting sound patterns.

\subsection{Information-theoretic Approach}

As an alternative to cope with the problems derived from the acoustic-correlates-only-approach, we pursue the view that linguistic activities 
are carried out as part of communication. The innovative proposal known as Information Theory was promulgated by Shannon and Weaver (1949). Before looking into its relevance to Korean vowel transitions in terms of contrast, let us define key concepts, which are going to be instrumental in the subsequent discussion, and then identify the mathematical equations to compute the roles of concerned concepts. To quantify the view of grammars as "the average bits per symbol to encode a certain message" as proposed by Shannon and Weaver, let us go over the crucial concepts one by one. Now, the information content of an individual element within a system is our first concern. Before providing a proper definition, our imminent task is to avoid a flurry of terms repeating the same concepts, recurrent in the previous literature: despite the pioneering attempt to accommodate phonological distinctive features in terms of information viewpoint, unfortunately, no name was given by Cherry, Halle and Jakobson (1952); information complexity is interchangeably used with plog (positive log probability) by Goldsmith (2002); surprisal or information content by Hume and Mailhot (2013), to name just a few. In this paper, referring to the information content of individual elements, the appellations, surprisal and information content, will be interchangeably used. Surprisal of an element $i$ is obtainable through the equation (1):

(1) $\mathrm{S}_{\mathrm{i}}=-\log _{2} \mathrm{p}_{\mathrm{i}}$

$S_{i}=$ surprisal of an element $i ; p_{i}=$ probability of an individual element $i$

As the metaphorical term, surprisal implies, Figure 4 makes it clear that the probability and the associated surprisal of an element are in inverse proportion. When the probability of an element is extremely low, its surprisal is maximized, and vice versa: 


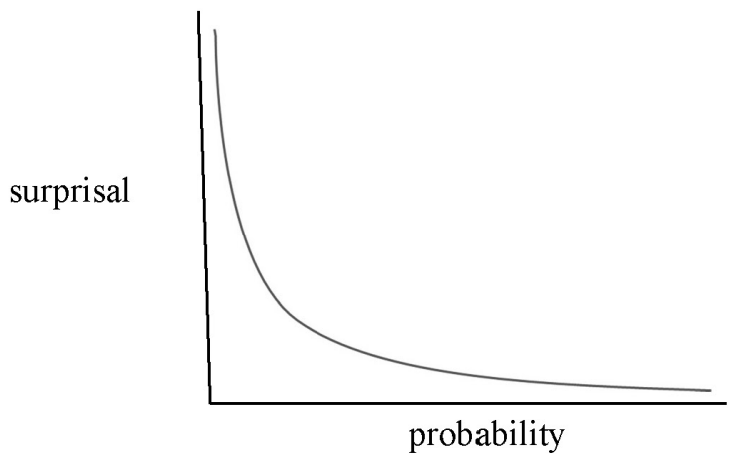

Figure 4. The interrelationships between the probability and surprisal of an element.

Next, the degree of communicative effectiveness of an element toward the whole system is referred to as entropic contribution $\left(\mathrm{H}_{\mathrm{i}}\right)$, and its measurements will be taken under the formula (2):

(2) $\mathrm{H}_{\mathrm{i}}=\mathrm{p}_{\mathrm{i}} * \log _{2} \mathrm{p}_{\mathrm{i}}$

$H_{i}=$ entropic contribution of an element $i ; p_{i}=$ probability of an individual element $i$

On the other hand, to compute amount of contrast between a pair of sounds, we employ the concept of relative contrastiveness, following Hume et al. (2011). The relative contrastivenss will be resorted to the comparison of the entropy value of a system when a merger takes place, with the preexisting entropy value before the occurrence of the merger. The calculation will be repeating over and over again until the overall set of potential mergers is exhausted. Relative contrastiveness (RC) of an element is calculated under (3):

(3) $\mathrm{RC}=\frac{\sum_{n \in M} \frac{\boldsymbol{H}_{\mathbf{1}}-\boldsymbol{H}_{2}}{H \mathbf{H}}}{|\mathrm{M}|}$ 
$R C=$ relative contrastiveness; $H_{1}=$ the entropy of the whole system without a merger; $\mathrm{H}_{2}=$ the entropy with a merger of concerned elements; $M=$ the set of all possible mergers; $|M|=$ the cardinality, i.e. the number of elements in the set $M$

What is more, we need to take perceptual distance into our consideration, which is to measure the distance among sounds viewed in light of perceptibility. It is expected that the more is the perceptual distance, the greater is the contrast among sound or the robustness of a sound distinction. To compute the perceptual distance between vowel pairs, we are going to rely on the equation (4), which is set up by Lindblom (1990):

$$
\mathrm{D}_{\mathrm{ij}}=\sqrt{\left(\mathrm{M} 1_{\mathrm{i}}-\mathrm{M} 1_{\mathrm{j}}\right)^{2}}+\left(\mathrm{M} 2_{\mathrm{i}}-\mathrm{M} 2_{\mathrm{j}}\right)^{2}+\left(\mathrm{M} 3_{\mathrm{i}}-\mathrm{M} 3_{\mathrm{j}}\right)^{2} \cdots
$$

$D_{i j}$ is the perceptual distance between two elements $i$ and $j M_{i}$ is $F 1$ of an element $i$ in Mel scale calculated under the formula $M=1127.01048 * \log _{e}$ $\left(1+\right.$ formant/700); the same is true to $M 2_{i,} M 3_{i}$, and the like.

\subsection{The probed corpus}

Korean National Corpus, which is our primary data sources, unofficially known as Sejong Corpus. It started to be built from 1997, and it is still an ongoing project. Let alone contemporary ordinary language, a great number of terms of professional fields and historical material are included in the plans. One of the tasks in tagging the collected data is related to a grammatical category eojeol, a phrasal word typical to Korean. Phrasal words stand for the ordinary words followed by particles or endings, if any. The boundary of phrasal words coincides with spacing in the written texts. The corpus is accessible through the website https://ithub. korean.go.kr. The statistics are given in Table 2: 
Table 2. Korean National Corpus by National Institute of the Korean Language

\begin{tabular}{|c|c|c|c|c|}
\hline $\begin{array}{c}\text { oral or } \\
\text { written text }\end{array}$ & size & $\begin{array}{l}\text { \#of phrasal words } \\
\quad \text { (as of 2011) }\end{array}$ & $\begin{array}{c}\text { \#of } \\
\text { phonemes }\end{array}$ & language \\
\hline written text & $\begin{array}{c}\text { project } \\
200,000,000 \\
\text { phrasal words }\end{array}$ & $\begin{array}{c}\text { raw } \\
63,899,412 \\
\text { grammatically tagged } \\
15,226,186 \\
\text { parsed tagged } \\
570,064 \\
\text { semantically tagged } \\
10,132,348 \\
\text { total } \\
89,830,015\end{array}$ & unknown & Korean \\
\hline
\end{tabular}

Further, we rely on B Yang (1996), H-Y Hwang and S-J Moon (2005) for Korean data sources, while Hillenbrand et al. (1995) serves our purpose to compare Korean and English vocalic properties.

\section{Results}

3.1. Disintegration of vowels $/ y /$ and /ø/

\subsubsection{Surprisal}

To diagnose the transitions of Korean vowel contrast, we are going to put the key concepts defined in Section 2.2 to test one by one from the Information-theoretic perspective. As a first attempt, let us start with examination of surprisal, associated with Korean simple vowels. The results of the investigation based on the randomly chosen six among the numerous files available in Korean National Corpus are given in Table 3 below: 
Table 3. Frequencies and surprisal of Korean vowels (the data from Korean National Corpus)

\begin{tabular}{c|c|c|c|c|c|c|c|c|c}
\hline \multicolumn{3}{|c|}{ overall } & \multicolumn{2}{c|}{ spoken } & \multicolumn{2}{c|}{ written } & \multicolumn{3}{c}{ surprisal } \\
\hline vowels & freq & prob & freq & prob & freq & prob & overall & spoken & written \\
\hline \hline $\mathrm{a}$ & 87744 & 0.2492 & 6804 & 0.2969 & 80940 & 0.2459 & 2.0046 & 1.172 & 2.239 \\
\hline$\varepsilon$ & 20450 & 0.0581 & 1478 & 0.0645 & 18972 & 0.0576 & 4.1053 & 3.9546 & 4.1178 \\
\hline $\mathrm{e}$ & 17789 & 0.0505 & 1174 & 0.0512 & 16615 & 0.0505 & 4.3076 & 4.2877 & 4.3076 \\
\hline$\Lambda$ & 40216 & 0.1142 & 3461 & 0.151 & 36755 & 0.1117 & 3.1304 & 2.7274 & 3.1623 \\
\hline o & 37285 & 0.1059 & 617 & 0.0269 & 36668 & 0.1114 & 3.2392 & 5.2163 & 3.1662 \\
\hline $\boldsymbol{\varnothing}$ & $\mathbf{5 4 9 1}$ & $\mathbf{0 . 0 1 5 6}$ & $\mathbf{2 4 4}$ & $\mathbf{0 . 0 1 0 6}$ & $\mathbf{5 2 4 7}$ & $\mathbf{0 . 0 1 5 9 4}$ & $\mathbf{6 . 0 0 2 3}$ & $\mathbf{6 . 5 5 9 8}$ & $\mathbf{5 . 9 7 4 8}$ \\
\hline $\mathrm{u}$ & 29746 & 0.0845 & 4896 & 0.0827 & 27850 & 0.0846 & 3.5649 & 3.596 & 3.5632 \\
\hline $\mathbf{y}$ & $\mathbf{2 2 1 7}$ & $\mathbf{0 . 0 0 6 3}$ & $\mathbf{9 7}$ & $\mathbf{0 . 0 0 0 3}$ & $\mathbf{2 1 2 0}$ & $\mathbf{0 . 0 0 6 4}$ & $\mathbf{7 . 3 1 0 4}$ & $\mathbf{1 1 . 7 0 2 8}$ & $\mathbf{7 . 2 8 7 7}$ \\
\hline$\dot{\mathrm{i}}$ & 48691 & 0.1383 & 3305 & 0.1442 & 45386 & 0.1397 & 2.8541 & 2.7939 & 2.8583 \\
\hline $\mathrm{i}$ & 62481 & 0.1774 & 3841 & 0.1676 & 58640 & 0.1781 & 2.4949 & 2.5769 & 2.4892 \\
\hline
\end{tabular}

What is really noticeable here is that both of the concerned two vowels /y/ and /ø/ have a particularly high surprisal, as highlighted in Table 3. It is obvious that their significantly low frequencies, regardless of spoken and written texts, contribute to the highest level of surprisal. The overall surprisal 6.0023 of $/ ø /$ and 7.3104 of $/ y /$ is not comparable to that of the rest of Korean vowels. What is special about vowels $/ y /$ and $/ \varnothing /$ becomes all the more evident through the graphic representation given in Figure 5:

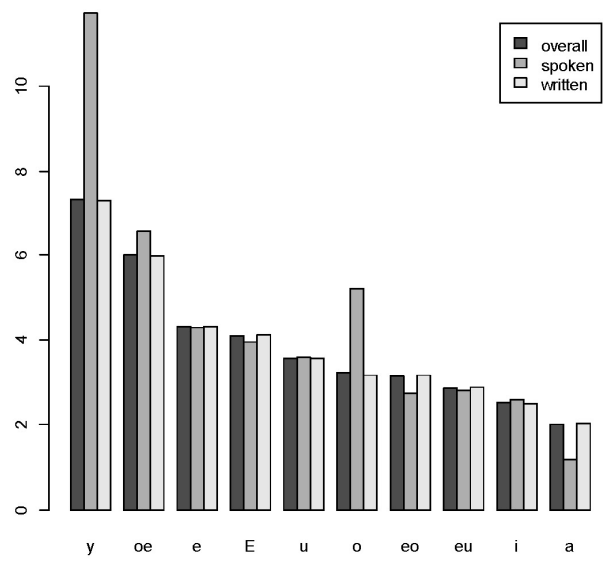

Figure 5. Surprisal (information content) of Korean vowels (n.b. Symbols $\mathrm{E}$, oe, eo and eu signify sounds $/ \varepsilon /, / \varnothing /, / \Lambda /$ and $/ \mathrm{i} /$, respectively). 
As will be elaborated in subsection 3.1.2 below, those with an extreme surprisal are easily subject to change, which is due to the low degree of entropic contribution. In terms of entropy, in other words, "the average bits per symbol to encode a certain message", those elements with a low entropic contribution are beneficial to increase the communicative efficiency when they transform themselves into another form or entirely disappear. In this regard, it would be safe to conclude that the property of a high surprisal might give a crucial momentum to compel the disintegration of Korean vowels $/ y /$ and $/ ø /$. Another thing to verify at this point is the hypothesis proposed by Hume and Mailhot (2011) that the phonological changes occurring to the elements with high surprisal should satisfy the condition of structure preservation (Kiparsky 1985), i.e. forbidding the illicit resultant structures in a given language. Here, notice that the outcomes of the change end up with the sequences /wi/ and /we/, which are definitely legal diphthongs in Korean. It is of no doubt that the disintegration of $/ y /$ and $/ \varnothing /$ obeys the requirement of structure preservation.

In conclusion, we claim that the properties of significantly high level of surprisal associated with two vowel pair /y/ and /ø/ contribute to their disintegration into diphthongs.

\subsubsection{Entropic contribution}

The corpus investigation brings us the outcomes as given in Table 4 and Figure 6. Opposite to what we observed in the case of surprisal, Table 4 shows us that the two vowels /y/ and /ø/ are extremely low in terms of their entropic contribution to Korean vowel system. The fact that regardless of spoken and written texts, resulted overall values 0.0936 and 0.0064 , as highlighted in Table 4, are recognizably minimized is all the more made clearer through Figure 6 below:

Table 4. Entropic contribution of Korean vowels (the data from Korean National Corpus)

\begin{tabular}{c|c|c|c}
\hline \multicolumn{4}{c}{ entropic contribution } \\
\hline \hline overall & spoken & written & surprisal \\
\hline \hline $\mathrm{a}$ & 0.4996 & 0.5231 & 0.5506 \\
\hline
\end{tabular}




\begin{tabular}{c|c|c|c}
\hline \multicolumn{5}{c}{ entropic contribution } \\
\hline \hline overall & spoken & written & surprisal \\
\hline$\varepsilon$ & 0.2386 & 0.2551 & 0.2371 \\
\hline $\mathrm{e}$ & 0.2175 & 0.2195 & 0.2175 \\
\hline$\Lambda$ & 0.3575 & 0.4118 & 0.3522 \\
\hline $\mathrm{o}$ & 0.343 & 0.1403 & 0.3527 \\
\hline $\boldsymbol{0}$ & $\mathbf{0 . 0 9 3 6}$ & $\mathbf{0 . 0 6 9 5}$ & $\mathbf{0 . 0 9 5 2}$ \\
\hline $\mathrm{u}$ & 0.3012 & 0.2974 & 0.3014 \\
\hline $\mathbf{y}$ & $\mathbf{0 . 0 4 6 1}$ & $\mathbf{0 . 0 0 3 5}$ & $\mathbf{0 . 0 4 6 6}$ \\
\hline$\dot{\mathrm{d}}$ & 0.3947 & 0.04029 & 0.3942 \\
\hline $\mathrm{i}$ & 0.4426 & 0.4319 & 0.4433 \\
\hline
\end{tabular}

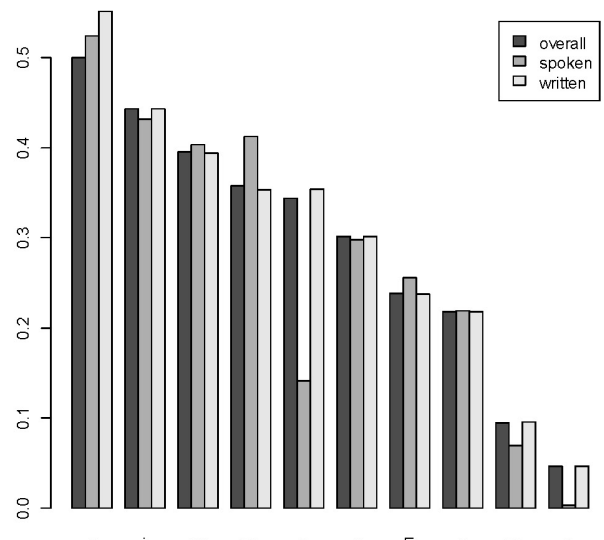

Figure 6. Entropic contribution of Korean vowels (n.b. Symbols eu, eo, $\mathrm{E}$ and oe signify $/ \dot{\mathrm{i}} /, / \Lambda / / \varepsilon /$, and $/ \varnothing /$, respectively).

By the nature of correlation between surprisal and entropic contribution that the elements posited at the extremes of too high or too low in terms of probability show extremity in terms of surprisal, the two extremes share a common ground that their entropic contribution is minimized. In other words, the elements with extreme probabilities are destined to contribute little to the entropy of the associated system, which is conceived to intervene as additional factor to instigate the relevant changes. What one's low contribution to the entropy of a system means is that the elements at stake so scarcely affect the total uncertainty of the system that some 
sort of changes is unnoticeable. Rather, the changes in themselves are likely to contribute to the increase of communicative efficiency. Coupled with the quality of high surprisal, the property of low entropic contribution of $/ y /$ and $/ \varnothing /$ is, therefore, believed to be crucially influential on their being left out of Korean simple vowel inventory.

To sum up what we have discussed in this section, the significant limitedness of $/ y /$ and $/ \varnothing /$ in terms of entropic contribution undermines the status quo of the concerned vowels would be held accountable for their disintegrations from monophthongs into diphthongs.

\subsubsection{Relative contrastiveness}

As introduced in section 2.2, to construe the property of a segment in light of relative contrastiveness, it is imperative to recalculate the entropy of a concerned new of system which undergoes a series of merger with that of a preexisting old system. The calculation keeps going on until all available segmental pairs are exhausted. The difference between the original entropy and recalculated one is believed to be momentous for deciding the amount of one's relative contrastiveness. To be more precise, the sum of the entropy differences between old and new systems divided by the entropy of the old system is to be divided again by the total number of concerned segmental pairs. On the basis of the selected database, we garnered the relative contrastivenss of Korean vowels, as given in Table 5 and Figure 7:

Table 5. Relative contrastiveness of Korean vowels (the data from Korean National Corpus)

\begin{tabular}{c|c}
\hline vowels & relative contrastiveness \\
\hline \hline $\mathrm{a}$ & 0.0846 \\
\hline$\varepsilon$ & 0.0396 \\
\hline $\mathrm{e}$ & 0.0434 \\
\hline$\Lambda$ & 0.0644 \\
\hline $\mathrm{o}$ & 0.0624 \\
\hline $\boldsymbol{D}$ & $\mathbf{0 . 0 2 1 3}$ \\
\hline
\end{tabular}




\begin{tabular}{c|c}
\hline vowels & relative contrastiveness \\
\hline \hline $\mathrm{u}$ & 0.0558 \\
\hline $\mathbf{y}$ & $\mathbf{0 . 0 1 1 8}$ \\
\hline$\dot{\mathrm{i}}$ & 0.069 \\
\hline $\mathrm{i}$ & 0.0762 \\
\hline
\end{tabular}

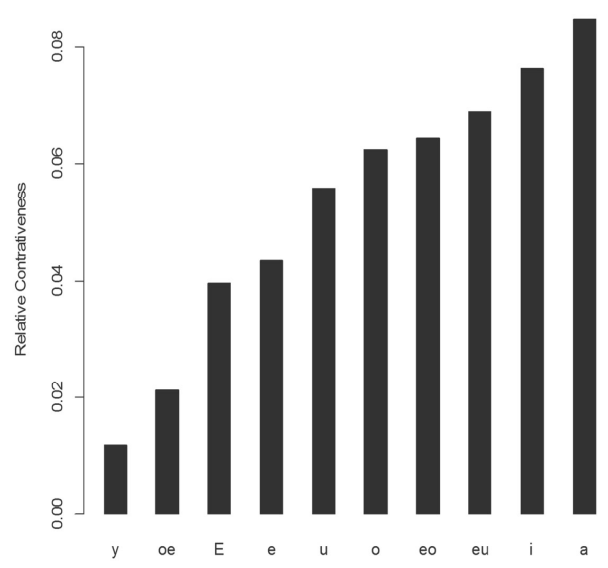

Figure 7. Relative contrastiveness of Korean vowels (data from Korean National Corpus)(n.b. Symbols oe, E, eo and eu signify /ø/, / $/, \Lambda /$ and $/ i /$, respectively).

The relative contrastiveness 0.0213 of /ø/ and 0.0118 of /y/, which are highlighted Table 5 and placed to the leftmost in Figure 7, prove to be the lowest among ten Korean simple vowels. Thus, it is evident that in parallel with surprisal and entropic contribution, the property of the weakest contrastiveness represented by the nature of the bottom-rankedness in Figure 7 contributes to the demise of two front rounded vowels /y/ and /ø/ from Korean vowel inventory.

\subsection{The $/ \mathrm{e} /-/ \varepsilon /$ merger: a perceptual view}

In section 1 , we mentioned that the front mid-vowel pair $/ e /-/ \varepsilon /$ is prone to neutralization cross-linguistically. However, in section 2.1, we noticed that it is imperative to be cautious not to seek what accelerates sound patterns entirely from fragmented individual acoustic properties 
of involved sounds. For instance, it is true that the formant frequencies $\mathrm{F} 1$ and F2 of Korean /e/ and / $/$ / jumble together, and they are acoustically mingling together, as Figure 8 shows:

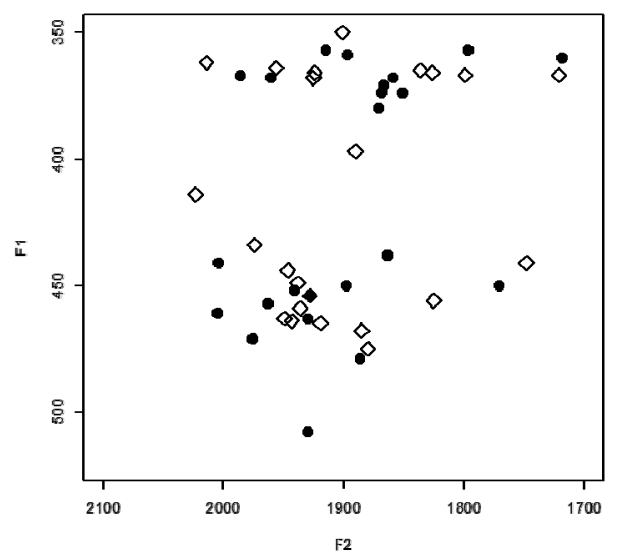

Figure 8. F1 and F2 Frequencies of Korean /e/ and $/ \varepsilon /$ (dark circles represent vowel /e; based on the measurements of H-Y Hwang and S-J Moon (2005).

What we want to propose here is that the acoustic properties are not the whole story of the vowel neutralization. What we are trying to do in this section is to explore the latent association of perceptual aspects with what happens here, instead of solely relying on regular formant frequencies of concerned two front mid-vowels.

As made explicit in section 2.2, the measurements of perceptual distance are attempted on the basis of acoustic formant frequencies of a vowel. For the purpose, we make use of the measurement of B Yang (1995), who tries to normalize the formant measurements, and thus, remove irrelevant variables like vocal track length and gender influence, etc. To convert the obtained regular formant frequencies into those of Mel psychoacoustic scale, the formula $M=1127.01048 * \log _{e}$ (1+formant/700) is employed. Ultimately, we come by those given in Table 6 and Figure 9: 
Table 6. Perceptual Distance Among Korean Vowels

\begin{tabular}{|c|c|c|c|}
\hline & \multicolumn{3}{|c|}{ perceptual distance } \\
\hline & male & female & overall \\
\hline the average of the rest & 411 & 495 & 449 \\
\hline A. $\Lambda-\dot{i}$ & 190 & 296 & 243 \\
\hline B. $0-\Lambda$ & 184 & 285 & 235 \\
\hline C. $a-\Lambda$ & 184 & 249 & 217 \\
\hline D. e-y & 168 & 265 & 217 \\
\hline E. $i-\varnothing$ & 167 & 245 & 206 \\
\hline F. $\varepsilon-\varnothing$ & 130 & 67 & 99 \\
\hline G. i-y & 112 & 81 & 97 \\
\hline H. e-ø & 94 & 74 & 84 \\
\hline I. $0-\mathbf{u}$ & 96 & 70 & 83 \\
\hline J. $\varepsilon-e$ & 117 & 39 & 78 \\
\hline
\end{tabular}

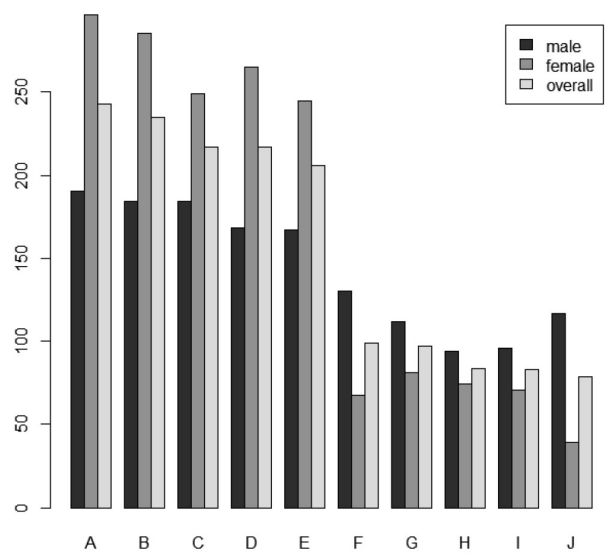

Figure 9. Perceptual distance among Korean vowels.

For the sake of saving space, among 45 available Korean simple vowel pairs, ten vowel pairs are presented here. As shown by the tabulation and illustration, it proves that the pair /e/-/E/ holds the shortest perceptual distance among the 45 possible Korean vowel pairs, and we can surmise that the tiny perceptual distance 78 , carried by the pair /e/-/ع/ would be responsible, partially or entirely, for the vowel merger. However, we must be cautious in our contention: the perceptual distance of the bottom 
three pairs: 78 of /e/-/ع/, 83 of /o/-/u/ and 84 of /e/-/ø/, as boldfaced in Table 6, clusters around 80 together. On the other hand, when focusing on female speakers, 39 of $/ \mathrm{e} /-/ \varepsilon /$ is recognizably lower than the rest of others. The vowel pair $/ \mathrm{e} /-/ \varnothing /$ is immediately eliminated from our attention, considering that $/ \varnothing /$ is on the brink of banishing. However, the pair $/ \mathrm{o} /-\mathrm{u} / \mathrm{is}$ quite bothering us. One thing to consider here is that the reliability of acoustic measurements of data source given by $\mathrm{B}$ Yang (1996). Regarding the pair /e/-/ع/, the rate of perceptual distance of male, 117 amounts to three times of female speakers (39), which is quite questionable. In spite of a moot point concerning the pair /o/-/u/ for its resistance against an expected merger, we can conjecture that the shortest perceptual distance of /e/ and / $/$ / among 45 vowel pairs and the unique vowel merger, to the exclusion of other vowel pairs, leads us to claim that perceptual distance among vowels exerts a certain amount of influence on the vowel merger one way or another.

\section{Concluding Remarks}

In this article, our endeavors have started from the premise that the weak contrast associated with concerned vocalic segments might bring about the wavering of what they sound like. At this juncture, the null hypothesis therein was that the correlates derived from a certain amount of information as a measure of communicative efficiency deeply impact the determination of the degree of contrast among phonological units. Specifically, focusing on a series of corpus investigations, what we found out boils down to the following points: Concerning Korean vowels /y/ and $/ \varnothing /$, the triad of surprisal, entropic contribution and relative contrastiveness serves the vowel disintegration in a positive way, without being seriously at odds with one another. However, by now, it is not entirely certain whether the three factors collaborate in a group in their exercise of momentum on what is taking place here or they work separately without intimate interactions.

For the issue of the merger of $/ \mathrm{e} /-/ \varepsilon /$, we shifted the gear to the view- 
point of perceptual distance. What we found is that perceptual distance seems to be the culprit in this change, but the resistance of the pair $/ \mathrm{o} /-/ \mathrm{u} /$ remains challenging to our solid conclusion, and for that reason, it is premature to take a strong position for the role of perceptual distance in the determination of the vowel merger.

One outstanding issue yet to be resolved is that the thresholds of extremes in surprisal or entropic contribution for triggering a certain type of sound changes should be redefined in a more rigorous way. For instance, what would be the tipping points for the diphthongization of simple vowels in light of surprisal or its reversal, entropic contribution? To be more precise, how much gap between $/ \mathrm{y} /$ and $/ \varnothing /$, and the remaining vowels is necessary to trigger or block the structure change? The elaboration of that point will contribute to reinforcing what we propose for the Korean vowel changes from the standpoint of Information Theory.

\section{References}

Cherry, E. Collin, Morris Halle, and Roman Jakobson. (1952). Toward the logical description of language in their phonemic aspect. Language 29, 34-46.

Goldsmith, John. (2002). Probabilistic models of grammar: phonology as information minimization. Phonological studies 5, 21-46.

Cruttenden, Alan. (2001). Gimson's pronunciation of English. London: Arnold.

Hall, Kathleen. (2009). A probabilistic model of phonological relationships from contrast to allophony. Ph.D. dissertation, Ohio State University.

Han, Yang-Gu. (2000). An experimental phonetic study on production and perception of English vowels by native Korean speakers. M.A. thesis, Wonkwang University.

Hillenbrand, James, Laura Getty, and Michael Clark. (1995). Acoustic characteristics of American English vowels. The journal of acoustic society of America 95, 3099-3111.

Hume, Elizabeth and Frédéric Mailhot. (2013). Distinctive features and Information Theory. Origins of sound changes: approaches to phonologization, ed. by Alan Yu, 29-47. Oxford: Oxford University Press.

Hume, Elizabeth, Kathleen Hall, Andrew Wedel, Adam Ussishkin, Martine Adda-Decker, and Cédric Gendrot. (2011). Anti-markedness patterns in French epenthesis: an Information-theoretic approach. Proceedings of the Berkeley 
Linguistic Society 37, 104-123.

Hwang, Hye-Young and Seung-Jae Moon. (2005). An acoustic study of Korean /e, $\varepsilon /$ and English $/ \varepsilon, æ /$ pronounced by Korean young male speakers. Speech sounds 56, 29-47.

Hur, Woong. (1965). Korean phonology. Seoul: Jeongeum.

Kiparsky, Paul. (1985). Some consequences of Lexical Phonology. Phonology 2, 85-138.

Labov, William, Sharon Ash, and Charles Boberg. (2006). The atlas of North American English: phonetics, phonology and sound change. Berlin: Walter de Gruyter.

LaCharité, Darlene and Carol Paradis. (2005). Category preservation and proximity versus phonetic approximation in loanword adaptation. Linguistic inquiry 36, 223-258.

Lindblom, Björn. (1990). Explaining phonetic variation: a sketch of the H \& H theory. Speech production and speech modeling, ed. by William J. Hardcastle and Alain Marchal, 403-439. Dordrecht: Kluwer.

Oh, Jeong-Ran. (1993). Modern Korean phonology. Seoul: Hyeongseol.

Shannon, Claude Elwood and Warren Weaver. (1949). The mathematical theory of communication. Urbana-Champaign: University of Illinois Press.

Shin, Ji-Young. (2000). Understanding speech sounds: solidifying the foundation of phonological research. Seoul: Hankookmunhwa.

Trubetzkoy, Nikolai Sergeyevich. (1939/1969). Principles of phonology. trans. by Christiane A.M. Baltaxe. Berkeley and Los Angeles: University of California Press.

Yang, Byunggon. (1996). A comparative study of American English and Korean vowels produced by male and female speakers. Journal of phonetics 24, 245-261.

Ponghyung Lee

Department of English Language and Literature

Daejeon University

Daehak-ro 62, Dong-gu, Daejeon City, 34520, South Korea

E-mail: phlee@dju.kr

Received: February 24, 2017

Revised version received: March 29, 2017

Accepted: April 16, 2017 
